Case Report

\title{
GLENOID LABRAL CYST PRESENTING WITH SUPRASCAPULAR NERVE PALSY
}

\author{
Siddharth M. Shetty ${ }^{1}$, Rajsankar N.R. ${ }^{2} \&$ B. Jayaprakash Shetty ${ }^{3}$ \\ ${ }^{1}$ Associate Professor, ${ }^{2}$ P.G. Student, ${ }^{3}$ Professor, Department of Orthopaedic Surgery \\ K.S. Hegde M edical Academy, Nitte University, M angalore - 575 018, Karnataka, India. \\ Correspondence: \\ Rajsankar N.R. \\ P.G. Student, Department of Orthopaedic Surgery, K. S. Hegde Medical Academy, \\ Nitte University, M angalore - 575 018, Karnataka, India. \\ Mobile : +919980951542 E-mail : rajsankar4029@yahoo.com
}

\section{Abstract:}

Introduction : Glenoid labral cyst in shoulder is an entity akin to meniscal cysts in knee. It usually manifests with features similar to impingement syndrome or rotator cuff weakness.

Clinical Picture : A 42yr old male patient with complaints of left shoulder pain and restriction of movement for 2 months with muscle wasting in left supraspinatus and infraspinatus region. M RI left shoulder showed partial tear of supraspinatus tendon, glenoid labral cyst in closed proximity and causing compression to suprascapular nerve with atrophy of infraspinatus muscle.

Treatment : Left shoulder diagnostic arthroscopy was done and followed with ganglion excision done through a posterior approach and suprascapular nerve was decompressed. Histopathology report showed features of an inflammatory cystic lesion.

Outcome : patient experienced resolution of symptoms within 4 months of surgery with significant restoration of rotator function and bulk.

Conclusion : Suprascapular nerve compressive neuropathy secondary to glenoid labral cyst is a rare entity but easily recognizable on a M RI scan and responds quickly to decompression.

Keyword: impingement, Suprascapular Nerve Compression

\section{Introduction:}

Shoulder pain is among the most common musculoskeletal complaints in the general population. Often, it is due to rotator cuff pathologies such as tendonitis and/or tears, labral pathologies such as superior labral anterior to posterior (SLAP) lesions or instability, capsular pathologies such as adhesive capsulitis, or articular pathologies such as acromioclavicular or glenohumeral arthritis. On rare occasions, suprascapular nerve compression may be the cause of such shoulder pain or weakness. Because of its rarity, this condition is unfortunately often not diagnosed until a magnetic resonance imaging (MRI) scan is performed on the patient who fails to respond to therapy.

\begin{tabular}{|c|}
\hline Access this article online \\
\hline Quick Response Code \\
\hline
\end{tabular}

cysts as a cause of supraspinatus nerve compressive neuropathy.

\section{Case Report :}

A 42yr old male shopkeeper presented to us with complaints of left shoulder pain with restriction of movement on overhead activity for 2 months. pain was insidious in onset and progressive dull in nature and moderate intensity it was noted around the back of the shoulder and extending to the proximal third of the arm over the lateral aspect there was no history of tingling sensation or radiation to the forearm and there was no history of trauma.

On examination there was hollowing of the supraspinatus and infraspinatus fossa with deltoid wasting. No local rise of temperature, non-tender, no swelling, scars or sinuses. Full range of motion of the shoulder passively but active abduction was upto 90 degree. The external rotators of the shoulder was weak 0 'Brien's test was negative. 
Routine haematological and biochemical investigation were normal. MRI left shoulder- cyst encrouching on the supraglenoid fossa with arising in close proximity to superior glenoid labral margin. Glenoid labral cyst in closed proximity and causing compression to suprascapular nerve with atrophy of infraspinatus muscle, with signal intensity changes noted in the supraspinatous tendon suggesting a partial tear. (Figure 1)
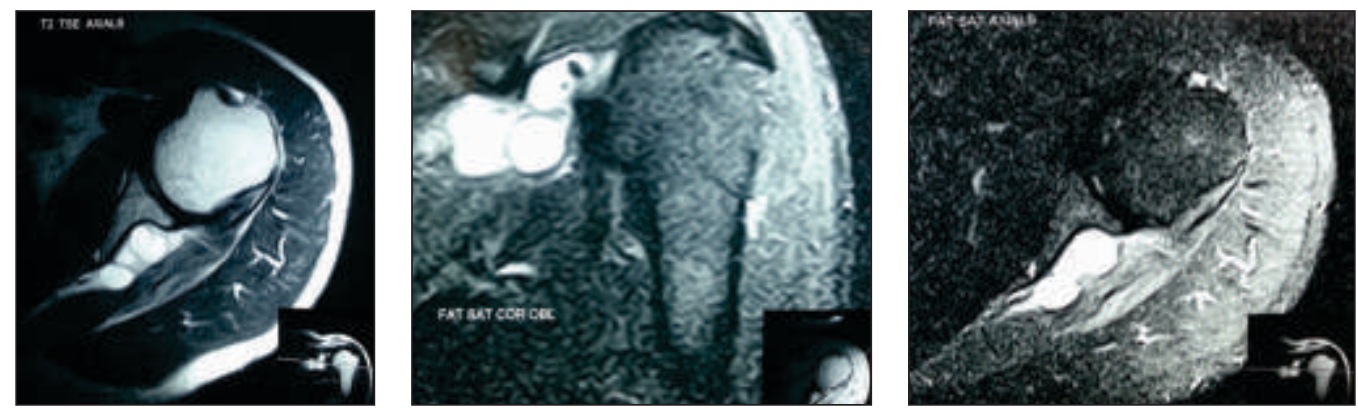

Figure 1: MRI left shoulder- cyst encrouching on the supraglenoid fossa with arising in close proximity to superior glenoid labral margin

Patient underwent shoulder arthroscopy in right lateral position and it revealed fraying of the under surface of the supraspinatus tendon and no tears, there was no evidence of any labral tears and the cyst was not clearly visualized. (FIGURE: 2A, 2B)

Figure 2: ( 2A, 2B) - Fraying of the under surface of the supraspinatus tendon and no tears, cyst was not clearly visualized.
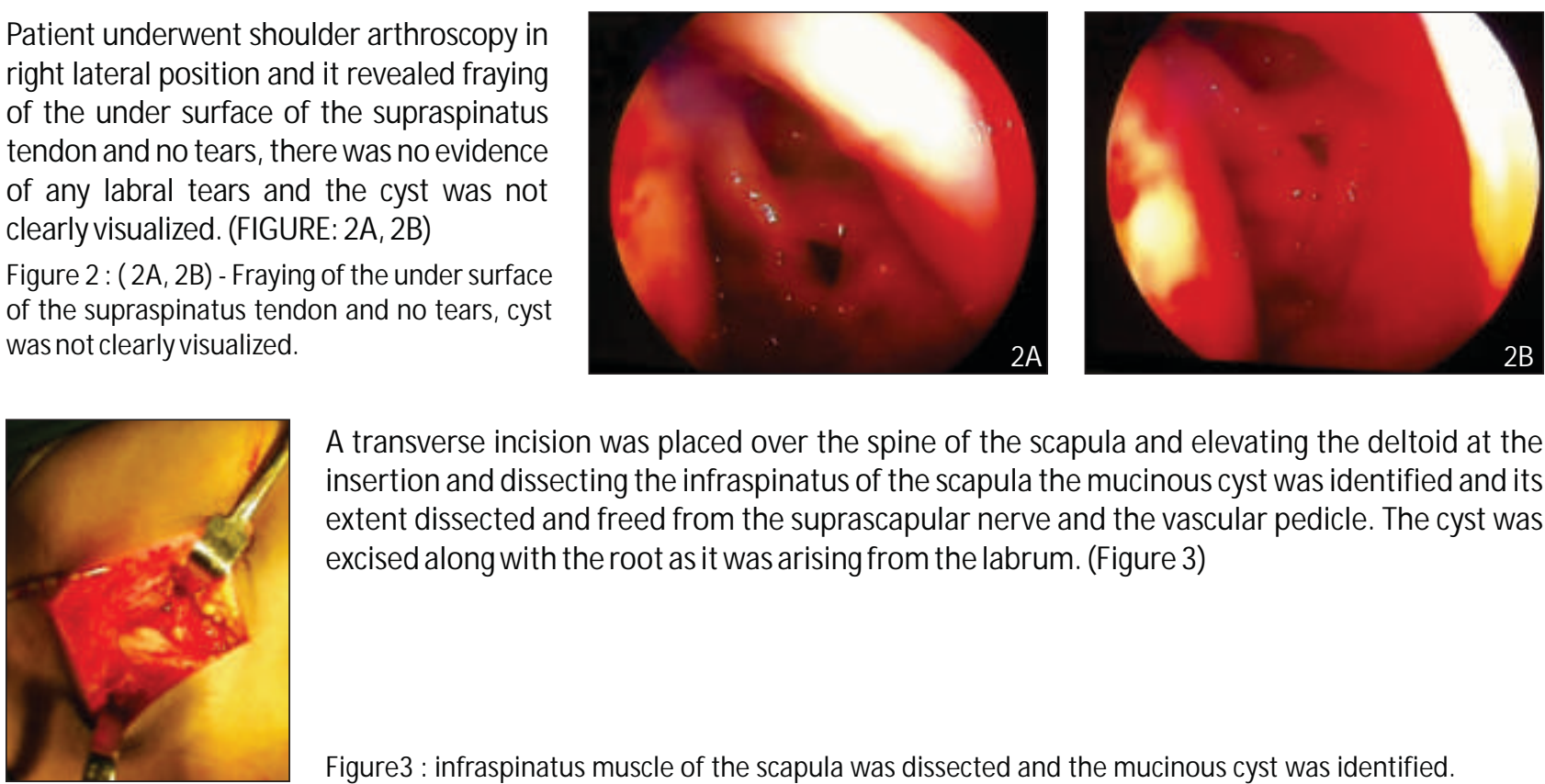

A transverse incision was placed over the spine of the scapula and elevating the deltoid at the insertion and dissecting the infraspinatus of the scapula the mucinous cyst was identified and its extent dissected and freed from the suprascapular nerve and the vascular pedicle. The cyst was excised along with the root as it was arising from the labrum. (Figure 3)

Figure3 : infraspinatus muscle of the scapula was dissected and the mucinous cyst was identified.

\section{Histopathology:}

The gross specimen showed greyish white mucinous content and the histopathological specimen showed inflammatory cystic lesion suggestive of a ganglion. (Figure 4)
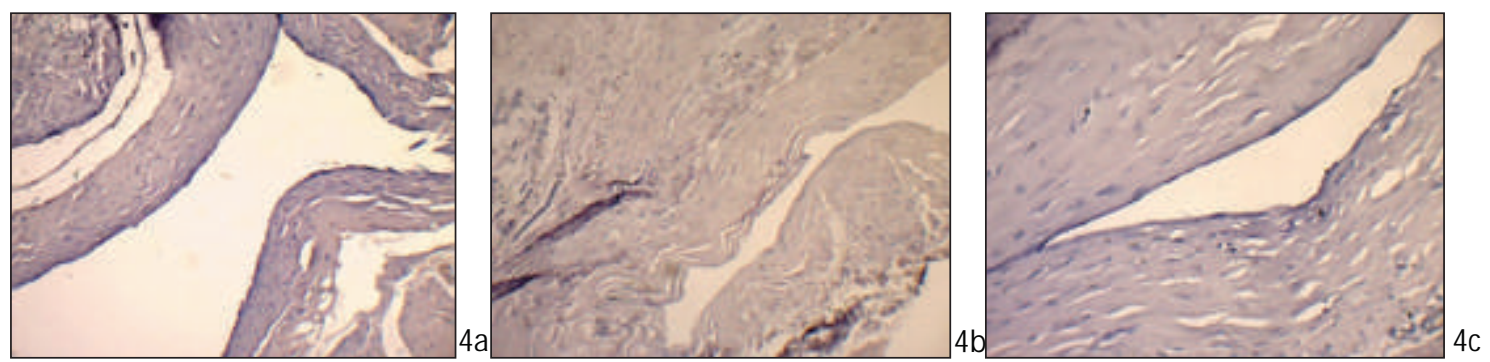

Figure $\mathbf{4 a}, \mathbf{4 b}, \mathbf{4 c}$ : inflammatory cystic lesion suggestive of a ganglion.

Postoperatively, she had slow resolution of symptoms over 4 months. By 6 months, he had complete resolution of symptoms and the muscle wasting had resolved. The operated shoulder was supported in

a sling for 10 days and they were encouraged to perform full range of active and passive movements immediately. 


\section{Discussion :}

The suprascapular nerve is a mixed peripheral nerve that arises from $\mathrm{C} 5$ and $\mathrm{C} 6$ roots with variable contribution from C4. The nerve runs to the suprascapular notch and lies in close relation to the posterior border of the clavicle. The suprascapular ligament forms the roof of the suprascapular notch, under which the nerve runs. The nerve then innervates the supraspinatus muscle as it enters the supraspinatus fossa, and receives sensory and proprioceptive branches from the glenohumeral and acromioclavicular joints, as well as the subacromial bursa and posterior aspect of the capsule. In up to $15 \%$ of individuals, the nerve also receives cutaneous afferents from the lateral deltoid. The nerve then runs inferolaterally where it wraps around the lateral margin of the scapular spine to pass through the spinoglenoid notch into the infraspinatus fossa, where it is a pure motor nerve supplying the infraspinatus muscle.

Suprascapular nerve entrapment as a case of shoulder dysfunction was first published in 1959 by Kopell $\mathrm{HP}^{3}$. Suprascapular nerve neuropathy was due to traction, trauma, infection or extrinsic compression by a spaceoccupying lesion, any space occupying lesion in and around spinoglenoid notch causes significant space constraints and compressive load of the nerve during overhead activities and throwing action.

The suprascapular nerve can be compressed by ganglion cysts, tumours or haematomas. As mentioned earlier, these ganglia are believed to develop when capsulolabral injuries create a valve-like effect and force synovial fluid into the surrounding tissues, similar to the way meniscal tears of the knee are believed to lead to meniscal cysts. Several authors have noted the high incidence of labral injuries with the presence of adjacent cysts. ${ }^{5-7}$

Patients with suprascapular neuropathy frequently present with pain and symptoms mimicking rotator cuff pathology. The pain is usually worse with overhead activities and it is also more common if the pathology is at the suprascapular notch rather than at the spinoglenoid notch. Muscle weakness usually manifests as weak external rotation and abduction of the arm. In chronic disease, there is often wasting of the infraspinatus as well as the supraspinatus if the pathology is at the suprascapular notch.

Treatment of suprascapular nerve entrapment of the shoulder consists of conservative or surgical options. Conservative management usually entails physiotherapy focused on range of motion and muscle strengthening exercises. The patients who fail to respond to conservative measures will need to be evaluated through M RI scan and confirm the diagnosis.

Surgical options include image guided decompression, but causes a risk of recurrence of up to $48 \%{ }^{4}$. Athroscopy of shoulder will be used to evaluate the state of labrum, rotator cuff, which can be combined with labral repair, decompression of cyst with cuff repair if required. Risk of recurrence when cyst decompression is combined with labral repair.

Open decompression is also a procedure of choice, direct visualization of the lesion and nerve decompression can be performed.

\section{Conclusion :}

Glenoid labral cyst in shoulder is an entity akin to meniscal cysts in knee. It usually manifests with features similar to impingement syndrome or rotator cuff weakness. Patients with failure of recovery of rotator cuff weakness when managed conservatively should be evaluated for suprascapular nerve neuropathy. Arthroscopic decompression is method of managing such lesions especially if associated with SLAP, combined with repair of SLAP. Excision of the cyst and decompression of the nerve by open procedure is a good alternative. 


\section{References :}

1. Bernard CS Lee, M uthukaruppan Yegappan, Palaniappan Thiagarajan. Suprascapular Nerve Neuropathy Secondary to Spinoglenoid Notch Ganglion Cyst , Ann Acad M ed Singapore ; December 2007, Vol. 36 No 1032-5

2. Chochole MH, Senker W, Meznik C, Breitenseher MJ. Glenoid-labral cyst entrapping the suprascapular nerve: dissolution after arthroscopic debridement of an extended SLAP Iesion. PubM ed, 1997 Dec;13(6):753-5

3. Kopell HP, Thompson WA. Pain and the frozen shoulder. Surg Gynecol Obstet 1959;109:92-6.

4. Aiello I, Serra G, Traina GC, Tugnoli V. Entrapment of the suprascapular nerve at the spinoglenoid notch. Ann Neurol 1982;12:314-6.

5. Ferretti A, Cerullo G, Russo GL. Suprascapular neuropathy in volleyball players. J Bone Joint Surg Am 1987;69:260-3.

6. Fehrman DA, Orwin JF, Jennings RM . Suprascapular nerve entrapment by ganglion cysts: a report of six cases with arthroscopic findings and review of the literature. Arthroscopy 1995;11:727-34.

7. Iannotti JP, Ramsey M L. Arthroscopic decompression of a ganglion cyst causing suprascapular nerve compression. Arthroscopy 1996;12:73945. 